\title{
ANÁLISIS DEL NIVEL DE COMPRENSIÓN LECTORA EN ESTUDIANTES DEL PROGRAMA ADMINISTRACIÓN DE EMPRESAS DE LA CUN
} Analysis of the reading comprehension level in students of the
Business Administration program at CUN

\section{Jenny Carolina López Guevara}

Docente, Facultad Administración de Empresas Corporación Unificada Nacional de Educación Superior CUN Correo electrónico: jenny lopez@cun.edu.co

\section{Yizeth Ramírez Velasco}

Docente, Facultad Administración de Empresas Corporación Unificada Nacional de Educación Superior CUN Correo electrónico: yizeth_ramirezvel@cun.edu.co

| Recibido/Received: 13/01/2021 • Aprobado/Approved: 29/06/2021 • Publicado/Published: 30/07/2021

\section{Resumen}

Esta investigación es de carácter cuantitativo con un enfoque descriptivo. En ella se analizó el nivel de comprensión de lectura de los estudiantes de primeros semestres, del programa Administración de la CUN. Se tomó una muestra de 308 estudiantes y 9 docentes, para un total de 317 participantes. Se implementó un muestreo de tipo probabilístico, a través de la aplicación de tres encuestas; dos (2) aplicadas a estudiantes y una aplicada (1) a docentes. Los resultados obtenidos permitieron identificar que los estudiantes de la muestra no desarrollaron competencias lectoras, en sus niveles de formación básica primaria y secundaria, por lo cual presentaron deficiencias en la decodificación de las palabras, en el dominio de las estrategias de comprensión, así como un escaso control y dirección del proceso lector. Adicional a ello, a partir de este estudio fue posible conocer, tanto el nivel de comprensión, como los hábitos y las técnicas de lectura, encontrando que las más utilizadas por los estudiantes, para fortalecer la comprensión lectora, fueron el subrayado, los resúmenes y los mapas conceptuales. También fue posible conocer la percepción que tuvieron los docentes, acerca del nivel de comprensión lectora de sus estudiantes, así como la manera de evaluar ésta en las clases; dichas variables, a su vez permitieron descubrir que a los encuestados les gustaría implementar actividades como olimpiadas, cursos teórico prácticos presenciales, clubes y talleres de lectura virtuales, en tanto encuentran que estas acciones permitirían mejorar sus niveles de comprensión lectora.

Palabras clave: comprensión lectora; lectura; estudiantes universitarios; niveles de comprensión lectora; dificultades de la comprensión lectora; habilidades lectoras..

\begin{abstract}
This research is quantitative with a descriptive approach, in which the level of reading comprehension of students in the first semesters of the CUN Administration program was analyzed, a sample of 308 students and 9 teachers was taken, for a total of 317 participants, probabilistic sampling was implemented through the application of three surveys, two (2) applied to students and one (1) to teachers. The results obtained allowed us to identify that the students in the sample did not develop reading skills in their levels of basic primary and secondary education, presenting deficiencies in word decoding, mastery of comprehension strategies, scarce
\end{abstract}

Lopez-Guevara, J. y Ramírez-Velasco, Y. (2021). Análisis del nivel de comprensión lectora en estudiantes del programa Administración de Empresas de la CUN. Revista FACCEA, Universidad de la Amazonia, Vol. 11(2), 126-137 pp. https://doi.org/10.47847/faccea.v11n2a2 
control and direction of the reading process. Additionally, it allowed to know the level of understanding, habits and techniques, highlighting that the most used by students to strengthen reading comprehension are underlining, summaries and concept maps. Also was possible to know the perception that teachers have about the level of reading comprehension of their students and the way to evaluate it in class, which allowed us to discover that the respondents would like the implementation of Olympics, theoretical practical face-to-face courses, virtual reading clubs and workshops to improve their reading comprehension levels.

Key words: reading comprehension; reading; college students; reading comprehension levels; reading comprehension difficulties; reading skills.

\section{INTRODUCCIÓN}

En la actualidad se ha hecho evidente que una de las falencias que presentan los estudiantes cuando inician su formación profesional reside en que no saben leer o no comprenden los textos y documentos que abordan, siendo este un factor determinante al momento de aprobar o de reprobar asignaturas.

Por esta razón, en esta investigación se realizó un análisis riguroso respecto de las diferentes etapas metodológicas, haciendo uso de tres instrumentos para la recolección de datos, lo cual permitió identificar las causas que dificultan la comprensión lectora en los estudiantes universitarios del nivel técnico, del programa Administración de Empresas en la Corporación Unificada Nacional de Educación Superior CUN. Así mismo, este estudio permitió sugerir estrategias 0 actividades que incidan en el mejoramiento de la comprensión lectora. Los resultados de dichos análisis se expondrán a lo largo del presente artículo.

Ahora bien, una vez realizada la revisión sobre los antecedentes se encuentra que existen diversos autores, a nivel de Colombia y de Latinoamérica, que se han referido a la comprensión lectora, como una problemática que afecta el desempeño escolar de los estudiantes. Respecto de lo anterior, a continuación, se refieren algunas investigaciones que reafirman este postulado.

En tal sentido, a nivel latinoamericano desde el Instituto Nacional de Estadística y Geografía INEGI de México, este, en su comunicado de prensa No. 190 de 2019 titulado "Población lectora en México con tendencia decreciente en los últimos cinco años" menciona lo siguiente:

Los resultados de la encuesta nacional del Módulo de Lectura -MOLEC- (2019) México, muestra, que el promedio de libros leídos por esta población es de 3,8 al año, mientras que en Chile se leen 5,4; en Argentina 4,6; en Colombia 4,1 y en Brasil 4,0. Considerando a la población que lee según su nivel educativo, se identifica que la mayor duración promedio de la sesión de lectura es mayor en el nivel de educación superior con 47' minutos. Según el motivo para la no lectura, se identifica que las razones principales son falta de tiempo (47,9\%), seguido por falta de interés $(21,7 \%)$. (p.1)

Por su parte, García \& Guevara (2017) concluyeron que los contenidos curriculares y el desarrollo académico que siguen los estudiantes, conforme avanzan en sus grados escolares, no parecen tener impacto sobre su 
comprensión ni sobre su motivación hacia la lectura de textos académicos (p.8). Del mismo modo, Palacios (2015) realizó un diagnóstico, con el objetivo de conocer las experiencias de los alumnos en relación con la lectura y evidenció que los estudiantes de la muestra no leen por hábito sino por obligación; además indicó que estos no se encuentran interesados en adquirir libros que les permitan fortalecer sus competencias académicas y lectoras y a su vez, presentan un bajo nivel de inferencia entre la información que contiene el título del texto, frente al resto del contenido (p.95).

En otro orden de ideas, para el caso de Bogotá, de acuerdo con un artículo publicado en 2016 por la revista Semana, el cual da cuenta del estudio realizado por la Universidad de la Sabana en conjunto con 12 instituciones de educación superior y que lleva por título "Nivel de lectura y escritura de los 'primíparos' es mediocre", se concluyó lo siguiente:

Los universitarios de primer y segundo semestre en Bogotá, tienen pobres y bajas habilidades en comprensión lectora; una de las razones que aporta el estudio para explicar esta situación es la ausencia de políticas institucionales en la educación media que aborde la enseñanza eficiente de las competencias en lectura y escritura de sus estudiantes. Debido a esto, los bachilleres no reciben las herramientas para manejar correctamente la lengua española; Por su parte, Mariano Lozano, profesor del departamento de lengua de la Sabana, determinó que "los estudiantes no saben escribir un ensayo, tienen mala ortografía, carecen de competencias en comprensión lectora y cuando deben enfrentarse al desarrollo de textos y lecturas de mayor complejidad o con sentido crítico (propio del nivel universitario), el déficit de los primíparos es notorio".(párr.3,6)

Los referentes antes mencionados aportan información de interés, acerca de las posibles causas que conducen a que los estudiantes presenten un bajo desempeño, frente a las actividades de lectura y de comprensión. Estas están relacionadas con la baja motivación e interés; con la imposición de los temas de lectura en el entorno educativo; con la ausencia de hábitos y cultura de lectura; con un bajo léxico y con un escaso análisis crítico, factores que también ocasionan dificultades en la redacción de textos. En concordancia con los antecedentes expuestos, igualmente se ratifica la importancia de generar la comparación, el análisis y la generación de estrategias, que permitan mejorar la comprensión lectora de los estudiantes universitarios, específicamente de los asociados al programa Administración de Empresas de la CUN.

Con base en lo mencionado, cabe resaltar que a lo largo de los años se han generado importantes cambios en la educación; con cada gobierno se establecen nuevas directrices y lineamientos que buscan alcanzar una "educación de calidad". Es por ello por lo que se hace necesario diseñar e implementar planes y proyectos, que apunten a la formación de estudiantes críticos y autónomos, que estén preparados para afrontar esta emergente sociedad de conocimiento y los cambios que lleva consigo la globalización. Partiendo de esta premisa, es claro que la lectura, la escritura y la oralidad (habla y escucha) son elementos claves y necesarios en la vida de las personas, pues es el lenguaje es una de las herramientas esenciales del ser humano, ya que además de incidir en el desarrollo de su pensamiento y en su capacidad para aprender, también influye en su desarrollo como ser social. 
Por su parte, a nivel institucional, partiendo del axioma de desarrollo del pensamiento lógico matemático y de la lectura crítica, la CUN entiende lo siguiente:

La lectura comprende la capacidad de sistematizar información de forma coherente, y estructurada, operando procesos analíticos en los que se usan lenguajes formales e informales. Estos procesos potencian competencias para la toma de decisiones en distintos ámbitos empresariales y de la vida cotidiana (PEC CUN, 2018, p.16).

Teniendo en cuenta lo anterior, esta investigación tomó trascendencia e importancia, en tanto se considera que, al identificar las posibles causas de las deficiencias en la comprensión lectora, de los estudiantes sujetos de estudio, es posible sugerir a la dirección del programa el diseño de actividades académicas y/o estrategias didácticas, que aporten al mejoramiento de las técnicas lectoras que dichos estudiantes poseen. Lo anterior, en función de que aquellos puedan captar fácilmente la idea principal de un texto, sin necesidad de leerlo varias veces. De esta manera, se mitiga el impacto negativo que genera la baja comprensión lectora, en el desempeño académico, laboral y social de los estudiantes.

\section{METODOLOGÍA}

Se abordó el nivel de comprensión lectora presentado en los estudiantes del programa de Administración de Empresas de la CUN del nivel técnico, desde la perspectiva académica, bajo el rol docente, apoyándonos en las evidencias obtenidas a través de la aplicación de los instrumentos investigativos y en los estudios y/o definiciones realizadas por diversos autores.

La investigación se realizó bajo una metodología cuantitativa, con alcance correlacional y con un enfoque de investigación-acción. Para ello, se aplicaron encuestas que, basadas en la medición numérica y en el análisis estadístico, permitieron comprobar las hipótesis y/o supuestos planteados, a través de la medición, la comparación y el análisis e interpretación de la información obtenida, acerca de las dificultades en la comprensión lectora de los estudiantes de primeros semestres, del programa administración de empresas de la CUN.

En cuanto al enfoque, este es de tipo investigación - acción, debido a que el mismo permitió entrelazar la metodología cuantitativa y el alcance correlacional, a la vez que combinar la teoría con la práctica, a través de la ejecución de un trabajo colaborativo, el cual permitió y facilitó la interacción entre las investigadoras y la población elegida. Para lo anterior, inicialmente se realizó un diagnóstico respecto del nivel de comprensión lectora que tienen los estudiantes del programa de Administración de Empresas (PAE) de la CUN, con el fin de analizar la incidencia que tiene la formación académica del PAE y la implementación de las nuevas herramientas tecnológicas, frente a las habilidades lectoras que desarrollan los estudiantes.

Como hipótesis se estableció que las estrategias implementadas por los docentes permiten a los estudiantes adquirir herramientas y fortalecer su nivel de comprensión lectora. También se definió que desde el programa 
de Administración de Empresas de la CUN se podrían implementar cursos, talleres y/o actividades, haciendo uso de las TIC's; dichos cursos permitirían a los estudiantes mejorar sus habilidades lectoras. Lo anterior, en la medida en la que el no desarrollo de competencias lectoras, en los niveles de formación básica primaria y secundaria, afecta la comprensión lectora de los estudiantes, durante la educación superior.

El ámbito de estudio comprendió estudiantes de primeros semestres $(1,2,3)$, del programa de Administración de Empresas de la Corporación Unificada Nacional de educación superior CUN, sede Bogotá centro, en la modalidad presencial.

El Tamaño de la muestra fue de 308 estudiantes y 9 docentes, para un total de 317 participantes. El tipo de muestreo fue probabilístico y la muestra estadística, debido a que se conoce la cantidad exacta de estudiantes del programa de Administración de Empresas en la modalidad presencial de la CUN de la sede centro, de la cual cada estudiante tiene la misma probabilidad de ser elegido. Con relación a los tipos de muestreo probabilístico se elige el aleatorio estratificado, considerando que se escogen grupos de estudiantes que cumplan con las características requeridas en el estudio. Las variables de análisis tenidas en cuenta para este estudio fueron las siguientes: principales dificultades en la comprensión lectora; nivel de comprensión lectora y acciones correctivas para mejorar los niveles de comprensión lectora.

Como técnica de investigación se eligió la encuesta. Para ello se diseñaron y se aplicaron tres (3) instrumentos: el número 1 (encuesta a estudiantes); el número 2 (medición de la comprensión lectora a los estudiantes); el número 3 (encuesta a docentes del programa de Administración de Empresas de la CUN). El análisis de los datos utilizado fue de tipo cuantitativo-descriptivo, a fin de confirmar las diferentes hipótesis planteadas; para ello, se aplicaron encuestas estructuradas a través de herramientas digitales; una vez estas fueron implementadas, se procedió con el proceso de recolección de datos, codificación, tabulación, elaboración de gráficas, medición numérica y análisis de los resultados. Estas acciones permitieron identificar las características lectoras de la muestra y establecer la correlación que presentan los estudiantes, entre su nivel de comprensión lectora, los hábitos lectores y las estrategias para la comprensión, que implementan en su formación académica.

\section{RESULTADOS}

A continuación se presentan los resultados más relevantes, obtenidos gracias a los tres (3) instrumentos de recolección de datos, lo cual permite evidenciar las técnicas y el nivel de comprensión lectora que presentan los estudiantes del Programa de Administración de Empresas de la CUN, a partir de su formación profesional. El tamaño de la muestra fue de 160 estudiantes para el instrumento $N^{\circ} 1$; de 148 para el instrumento $N^{\circ} 2$ y de 9 docentes para el instrumento $\mathrm{N}^{\circ} 3$. En el siguiente numeral se presentarán los resultados obtenidos.

\section{Instrumento $N^{\circ}$.1: encuesta a estudiantes}

El objetivo de este instrumento fue conocer la opinión y/o la experiencia de los estudiantes, frente a la "comprensión de textos"; así mismo, identificar las estrategias utilizadas por los estudiantes para comprender 
los mismos; también se quiso conocer el tipo de textos que aquellos leen, la frecuencia de lectura, el tipo de lector y las sugerencias para la implementación de mecanismos que favorezcan la comprensión lectora en la CUN.

De acuerdo con los resultados obtenidos, se determina que el 51,2\% de los estudiantes manifiestan ser buenos lectores; el 43,8\% regulares; el 1,9\% malos y únicamente el 3,1\% se consideran excelentes lectores (Tabla 1). De acuerdo con lo anterior, se concluye que es necesario implementar estrategias de motivación lectora, que permitan mejorar dichas competencias, con el fin de incrementar el número de estudiantes que se consideren a sí mismos como excelentes lectores.

En cuanto a la pregunta sobre si los estudiantes comprenden fácilmente la idea principal y la temática que aborda el texto, del total de los encuestados (125) el 78.1\% manifestaron que sí comprenden fácilmente la idea principal, frente a (35) 21,9\% que dicen no comprender con facilidad.

Por otra parte, al indagar el número de veces que un estudiante debe leer un texto para entenderlo, se identificó que el 76,3\% de los estudiantes debe leer como mínimo dos (2) veces un texto para comprenderlo, mientras que el $13,8 \%$ manifestó necesitar sólo una (1) lectura, incluso el 10\% manifestó requerir más de tres (3) lecturas, para lograr su comprensión. De otra parte teniendo en cuenta la variable sobre la comprensión del significado de la mayoría de palabras en la lectura de un texto, 132 estudiantes dicen entender el significado de estas, mientras que 28 arguyen no comprenderlo.

Según los resultados obtenidos con la pregunta anterior, el $82,5 \%$ de los encuestados manifiesta que comprende el significado de las palabras cuando lee, lo que evidencia que la mayor parte de los estudiantes manejan un buen vocabulario (Tabla 1 y 2 ).

Tabla 1.

Consideración frente a ser lector.

\begin{tabular}{lc}
\hline Opciones de Respuesta & Cantidad de estudiantes \\
\hline Excelente & 5 \\
Bueno & 82 \\
Regular & 70 \\
Malo & 3 \\
\hline
\end{tabular}

Fuente: elaboración propia.
Tabla 2.

Número de veces que se debe leer un texto académico o literario para comprenderlo.

\begin{tabular}{lc}
\hline Opciones de Respuesta & Cantidad de estudiantes \\
\hline Una (1) vez & 2 \\
Dos (2) veces & 122 \\
Tres (3) veces & 15 \\
Cuatro (4) veces o más & 1 \\
\hline
\end{tabular}

Fuente: elaboración propia.

Al analizar los resultados obtenidos en las preguntas, observamos que el 78,1\% de los estudiantes afirma comprender fácilmente la idea principal de los textos que lee. Sin embargo, se evidencia que estos deben leer más de dos veces un texto, para comprender la idea principal y el significado de las palabras, lo que muestra una aparente contradicción, frente al nivel de comprensión lectora, al no haber concordancia en las respuestas. 
De igual modo, los encuestados señalan que leen en promedio dos veces a la semana (Tabla 3), por lo que se puede inferir que los hacen de acuerdo con sus obligaciones académicas, pero no por gusto personal. Lo anterior, demuestra poca motivación lectora y, por lo tanto, un entendimiento básico de los textos.

Frente a las estrategias que implementan los estudiantes para leer y comprender un texto, se encuentra que la más desarrollada es el subrayado, dado que 99 de los encuestados la utiliza, frente a 17 que dice utilizar el resumen y, en menor proporción, el mapa conceptual. Respecto de esta última herramienta, tan solo 5 estudiantes dicen aplicarla.

De acuerdo con los resultados obtenidos se puede observar que, si bien los estudiantes suelen utilizar diferentes estrategias para comprender un texto, como ya se ha mencionado antes, la mayor parte de ellos utiliza la técnica del subrayado, la cual permite resaltar las partes del texto que consideran más importantes; lo anterior, con la finalidad de visualizar rápidamente las ideas o conceptos principales que encuentran tras la lectura.

Tabla 3.

Número de veces de lectura de textos a la semana.

\begin{tabular}{lc}
\hline Opciones de Respuesta & Cantidad de estudiantes \\
\hline Una (1) vez & 41 \\
Dos (2) veces & 59 \\
Tres (3) veces & 49 \\
Cuatro (4) veces o más & 11
\end{tabular}

Fuente: elaboración propia.
Figura 1.

Estrategias para leer y comprender un texto.

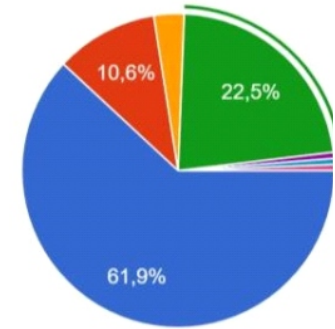

Por otra parte, en cuanto a la inclinación de los estudiantes para que a nivel institucional se implementen estrategias, a fin de mejorar los niveles de comprensión lectora se presentan los siguientes resultados (Tabla 4).

En tal sentido, del total de los estudiantes encuestados se evidenció que la mayoría de estos, opinan que la institución de educación superior debería implementar actividades que incentiven la lectura. Entre estas

Tabla 4.

Estrategias a nivel institucional, para mejorar los niveles de comprensión lectora en los estudiantes, según la información ofrecida por estos.

\begin{tabular}{lc}
\hline Opciones de Respuesta & Cantidad de estudiantes \\
\hline Talleres de lectura virtuales & 39 \\
Implementación de un curso teórico práctico presencial en comprensión lectora & 86 \\
Establecer una lista de libros a leer por asignatura & 31 \\
Otras & 4 \\
\hline
\end{tabular}

Fuente: elaboración propia. 
actividades destacan la realización de olimpiadas lectoras y la implementación de un curso teórico-práctico presencial, que les permita adquirir herramientas, a partir de las cuales mejoren su nivel de comprensión lectora.

Ahora bien, frente a su formación escolar (primaria o secundaria), se puede determinar que el $73,8 \%$ de los estudiantes manifiesta que, en su formación básica y secundaria, no les brindaron técnicas de comprensión lectora. Este es un dato importante para el desarrollo de este proyecto, debido a que permite confirmar la hipótesis planteada, a partir de la que se expone que los estudiantes no desarrollaron competencias lectoras, en los niveles de formación básica primaria y secundaria, lo cual afecta su comprensión lectora en la educación superior.

\section{Instrumento $N^{\circ}$ 2: Medición de la comprensión lectora en estudiantes universitarios}

El instrumento presenta un artículo denominado ¿Qué impacto tendrá la IA en el espacio de trabajo? Este fue leído por los estudiantes, quienes dieron respuesta a 11 preguntas relacionadas con el artículo propuesto. El objetivo de este instrumento consistió en evaluar el nivel de comprensión lectora que tuvieron los estudiantes al leer ese artículo, que trataba sobre la inteligencia artificial; adicional a ello, esta actividad permitió evidenciar la validez de las respuestas dadas por los estudiantes, a las preguntas del instrumento $N^{\circ} 1$.

Una vez aplicado el instrumento y analizados sus resultados, se comprobó que los estudiantes del programa Administración de Empresas de la CUN, del ciclo técnico, poseen un nivel de comprensión lectora medio/regular, mostrando una mayor dificultad en las preguntas que requieren de un análisis crítico, así como de ejercicios de argumentación, generación de asociaciones o inferencias. En tal sentido, el 87,6\% de los estudiantes respondió de manera incorrecta las preguntas en las cuales se requería un mayor grado de comprensión lectora, denotando que tan sólo el 12,4\% de los mismos, lograron identificar la idea principal del texto, reconocieron el vocabulario y realizaron una lectura reflexiva y crítica del texto. Lo anterior permite concluir que los estudiantes no se concentran para realizar la lectura, que realizan lecturas superficiales, que no implementan estrategias para la comprensión lectora y que en su mayoría dan respuestas "obvias" a preguntas que tienen un menor nivel de dificultad.

\section{Instrumento $N^{\circ}$ 3: Encuesta a docentes del programa Administración de Empresas de la CUN.}

Esta encuesta estuvo destinada a conocer la forma en que los docentes evidenciaron y evaluaron la comprensión lectora en sus estudiantes. Teniendo en cuenta la percepción de los docentes se encuentra una correlación directa con la conclusión general emitida para instrumento $\mathrm{N}^{\circ} 2$, en la cual se menciona que los estudiantes presentan un nivel de comprensión lectora medio/regular, conclusión que se contrasta con la percepción de los mismos estudiantes, quienes manifiestan tener un nivel bueno/regular.

Los docentes de la CUN que fueron encuestados dicen implementar diversas estrategias para promover la lectura en sus estudiantes. Dichas estrategias son por ejemplo: el análisis de textos mediante la socialización en clase; el desarrollo de mapas conceptuales e infografías y el uso de herramientas digitales. 
Posterior a la aplicación de los tres (3) instrumentos de recolección de datos, con los cuales se identificó el nivel de comprensión lectora y el uso de estrategias didácticas por parte de los estudiantes del programa de Administración de Empresas de la CUN, se procedió al análisis y la revisión de cada una de las preguntas aplicadas y de sus respectivos hallazgos. Gracias a este análisis se evidenció que los factores que inciden de manera desfavorable en el nivel de comprensión lectora de los estudiantes, se encuentran relacionados con deficiencias en la decodificación, con problemas de memoria, con falta de dominio de las estrategias de comprensión y con escaso control y dirección del proceso lector, lo que conlleva a determinar que los estudiantes del PAE presentan un nivel de comprensión lectora catalogado como medio/regular.

Por otra parte, se determinó que las estrategias adecuadas para identificar si los estudiantes comprenden una lectura son las siguientes: retroalimentación en grupo en una mesa redonda, de acuerdo con el análisis y con las condiciones y si en verdad lo articulan con su proyecto de vida; sintetizar la información; asociación con casos reales; aplicación de la lectura para solución de problemas y estrategias para saber si el estudiante maneja el tema de la lectura y lo expone con claridad. A su vez, se observa que los docentes aplican diferentes estrategias para evaluar la comprensión lectora de sus estudiantes, tomando como base la realización de diversas preguntas, a través de acciones como debates, evaluaciones, talleres, foros, entre otras actividades.

Finalmente, con los hallazgos obtenidos se logró visibilizar las diversas técnicas utilizadas por los estudiantes y por los docentes de la institución, para fortalecer la comprensión lectora; estas son el subrayado, los resúmenes, los mapas conceptuales, los foros, gráficos y las rondas de preguntas entre otros.

Tabla 5.

Nivel de lectura de los estudiantes.

\begin{tabular}{lc}
\hline Opciones de Respuesta & Cantidad de docentes \\
\hline Alto & 0 \\
Medio & 6 \\
Bajo & 3 \\
\hline
\end{tabular}

Fuente: elaboración propia.
Figura 2.

Porcentaje de nivel de lectura de los estudiantes.

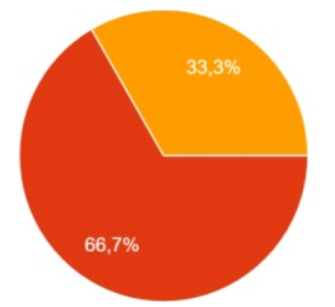

Alto

Medio

Bajo

Tabla 6.

Recursos didácticos implementados con los estudiantes para promover la lectura.

\begin{tabular}{lc}
\hline Opciones de Respuesta & Cantidad de docentes \\
\hline Creación de actividades lúdicas de un tema en particular & 1 \\
Blogs, vídeos, multimedia & 1 \\
Herramientas virtuales, redes sociales & 1 \\
Lecturas y análisis de textos, mediante socialización en clase & 1 \\
Los cuentos y parábolas & 1 \\
Desarrollo de mapas conceptuales e infografías & 1 \\
Juegos & 1 \\
\hline
\end{tabular}

Fuente: elaboración propia. 
También fue posible conocer las sugerencias acerca de las diferentes actividades que pueden implementarse, con el fin de mejorar los niveles de comprensión lectora en el programa, tales como olimpiadas, cursos teórico-prácticos presenciales, clubes de lectura y talleres de lectura virtuales, entre otros recursos.

\section{CONCLUSIONES}

Una vez aplicados los instrumentos y realizado el correspondiente análisis, se pudo determinar que los estudiantes objeto del estudio presentan un nivel de comprensión lectora regular, debido a que sólo realizan lecturas bajo el contexto formativo en el que se encuentran y no por una afición o gusto propio. Lo anterior produce que, contrario a lo que los estudiantes consideran sobre su propio nivel lector, el cual en general determinan como bueno, en la práctica se evidencie lo contrario. Ello, en tanto se encuentra que estos no logran comprender totalmente los textos sugeridos; además, presentan escasez de vocabulario y no utilizan de manera adecuada las técnicas más comunes y disponibles para comprender textos, tales como el subrayado, los resúmenes y los mapas conceptuales, entre otras herramientas.

Se evidencia además que la mayor dificultad que presentan los estudiantes para comprender textos tiene que ver con el escaso vocabulario que demuestran; igualmente, no dominan el proceso de memorización (memoria a corto plazo); tampoco muestran tener gusto por la lectura y consideran como actividad lectora únicamente al proceso de "leer" textos sueltos en internet y en redes sociales. Estos aspectos, producen que la calidad en la presentación de evaluaciones escritas y/u orales, en las exposiciones y en el ejercicio de actividades que impliquen argumentación de sus propias ideas se dificulte

Por otra parte, la mayoría de los estudiantes considera importante que la universidad implemente actividades que incentiven la lectura, tales como olimpiadas lectoras, cursos teórico-práctico presenciales, talleres de lectura virtual y un club de lectura; ello, con la intención de que dichas actividades les permitan adquirir herramientas y técnicas, para mejorar su nivel de comprensión lectora.

A su vez, dando respuesta a las hipótesis planteadas, se entiende que es importante partir de los antecedentes académicos de los estudiantes. Respecto de estos, se encuentra que la mayor parte de la muestra manifiesta que en su formación básica primaria y secundaria, no le brindaron técnicas de comprensión lectora o, en su defecto, que el estudiante no apropió las herramientas y por ende no las aplicó durante los siguientes niveles de su proceso educativo; lo anterior, a su vez ha generado una falencia o un déficit en el momento de cursar su pregrado, ya que el nivel de comprensión lectora que se requiere en la universidad es alto, comparado con los otros niveles formativos señalados.

También es importante señalar que, frente a la opinión de los docentes participantes, se identificó que las estrategias implementadas con mayor frecuencia, para evaluar la comprensión lectora de sus estudiantes y la comprobación de sus conocimientos, es a través de la participación en clase, del desarrollo de mapas conceptuales, de las infografías y del uso de TIC's. Al respecto, Carrillo (2017) afirma que es importante 
enseñar a los estudiantes a comprender lo que están leyendo y a explicar con sus propias palabras qué fue lo que entendieron a través de la lectura. (p.4)

De igual modo, las estrategias y las herramientas implementadas por los docentes permiten que los estudiantes conozcan y apliquen estrategias de comprensión lectora, que los conlleven a mejorar o ampliar su vocabulario, a decodificar palabras, a interpretar textos, a extraer ideas y a desarrollar un pensamiento reflexivo. Sin embargo, estas técnicas suelen ser utilizadas únicamente en los espacios académicos, en los cuales hay acompañamiento por parte del docente y, por tanto, en el trabajo autónomo el estudiante las olvida o sencillamente no las utiliza, lo que impide que el nivel de comprensión lectora mejore.

Para finalizar, es importante recalcar la necesidad de que, desde la dirección del programa, se desarrollen actividades de lectura entretenida, que contribuyan a generar una cultura lectora y que además rompan con el paradigma a partir del cual se piensa que la lectura es aburrida. Lo anterior, con el fin de que, a través de dichas actividades, se pueda obtener como resultado un incremento en el porcentaje de estudiantes que se consideran excelentes lectores, lo que contribuirá no sólo en la formación del estudiante, sino que a nivel institucional se podrá evidenciar en los resultados que se obtengan en las pruebas Saber T\&Ty Saber Pro.

\section{Recomendaciones}

En pro del mejoramiento en los niveles de comprensión lectora de los estudiantes se sugiere a la Corporación Unificada Nacional de Educación Superior CUN y específicamente al programa Administración de Empresas lo siguiente:

$\checkmark$ Elaborar una cartilla en la cual se recopilen las diversas técnicas y herramientas que pueden implementar los estudiantes, para comprender textos y mejorar su rendimiento académico.

$\checkmark$ Durante la semana de inducción implementar evaluaciones diagnósticas, que permitan conocer el nivel de comprensión lectora que presentan los estudiantes, antes de iniciar su proceso de formación profesional.

$\checkmark$ Con base en los resultados evidenciados en la prueba diagnóstica mencionada se recomienda desarrollar un curso de fortalecimiento de las habilidades lectoras, a través de herramientas lúdicas, innovadoras y creativas, a partir de las cuales se incentive el gusto por la lectura.

$\checkmark$ Incluir e incentivar la lectura de textos literarios complementarios al proceso de formación, como una actividad que permita al estudiante desarrollar, mejorar y explotar sus habilidades lectoras, con el acompañamiento activo de los docentes. Esta actividad puede ejecutarse eligiendo una lista de libros guía o base para "leer" por semestre; la misma será evaluada en diferentes momentos y asignaturas.

$\checkmark$ Se recomienda realizar un muestreo a nivel global en la institución, el cual permita conocer el nivel de comprensión lectora de toda la población estudiantil. 


\section{REFERENCIAS BIBLIOGRÁFICAS}

Alcaldía Mayor de Bogotá. (2016) Plan distrital de Lectura. Leer es Volar. Alcaldía Mayor de Bogotá. https://bogota.gov.co/mi-ciudad/cultura-deporte-y-recreacion/bogota-lanza-el-plan-distrital- delectura-y-escritura-leer

Carrillo, M. V. (2017). La lectura y su importancia en la adolescencia. Vida Científica Boletín Científico de la Escuela Preparatoria, 4(5), 10.

García, J., \& Guevara B, C. (2017). Variables académicas, comprensión lectora, estrategias y motivación en estudiantes universitarios. Revista electrónica de investigación educativa, 19(2), 78-90. http://www.scielo.org.mx/scielo.php?pid=S1607- 40412017000200078\&script=sci arttext.

García, A. D., \& Ortiz, L. Á. (2010). Procesos de lectura en estudiantes universitarios. Paideia Surcolombiana, (15), 134-142. https://doi.org/10.25054/01240307.1097

Instituto Nacional de Estadística y Geografía INEGI. (2019). Módulo sobre Lectura (MOLEC). INEGI. https://www.inegi.org.mx/contenidos/saladeprensa/boletines/2019/EstSociodemo/MOLEC20 18 04.pdf

Palacios E, L.S., (2015).Mejoramiento de la comprensión lectora en alumnos de segundo semestre del programa de español y Literatura de la Universidad Tecnológica del Chocó Diego Luis Córdoba [Tesis de maestría, Universidad Nacional de Colombia] http://bdigital.unal.edu.co/50602/

Corporación Unificada Nacional de Educación Superior, CUN. (2018) Plan Educativo Cunista (PEC). CUN

Semana. (2016). Nivel de lectura y escritura de los 'primíparos' es mediocre. Semana. https://www.semana.com/educacion/articulo/universidad-de-la-sabana-estudio-muestra-que- elnivel-de-escritura-y-lectura-de-los-universitarios-colombianos-es-mediocre/460982 